\title{
Componentes oculares em anisometropia
}

\author{
Theocularcomponents in anisometropia
}

\author{
David Tayah ${ }^{1}$ \\ Vinicius Coral-Ghanem² \\ Milton Ruiz Alves ${ }^{3}$
}

\begin{tabular}{|l|}
\hline RESUMO \\
\hline Objetivo: Comparar as correlações dos componentes oculares (compri- \\
mento axial, comprimento do segmento anterior, poder médio da córnea, \\
profundidade da câmara vítrea e poder refrativo equivalente) com o erro \\
refrativo total do olho portador da menor e da maior ametropia em aniso- \\
métropes. Métodos: Foi realizado um "survey" analítico conduzido em \\
população de 68 anisométropes de duas ou mais dioptrias atendida no \\
Ambulatório da Clinica Oftalmológica do Hospital das Clínicas da Facul- \\
dade de Medicina da Universidade de São Paulo Os anisométropes foram \\
submetidos àrefração estática objetiva e subjetiva, ceratometria e biometria \\
ultra-sônica. Resultados: Não houve diferença significativa entre os \\
valores dos componentes oculares medidos dos olhos portadores da \\
menor e da maior ametropia. Os olhos portadores da menor ametropia \\
apresentaram as mesmas correlações significantes observadas em olhos \\
emétropes, ou seja, correlação da refração com comprimento do segmento \\
anteriore comprimento axial, e correlação do comprimento axial compoder \\
corneano e profundidade da câmara vítrea. Os olhos portadores da maior \\
ametropia apresentaram correlação significante da refração com o compri- \\
mento axial e do comprimento axial com a profundidade da câmara vítrea. \\
Ainda em ambos os olhos observou-se correlação significante do poder \\
do cristalino com a profundidade da câmara anterior. Conclusão: Os olhos \\
portadores da menor ametropia desenvolveram as correlações mais fre- \\
qüentemente observadas nos olhos emétropes. Os olhos portadores da \\
maior ametropia não desenvolveramas mesmas correlações dos emétropes. \\
\hline
\end{tabular}

Descritores: Anisometropia; Biometria/métodos; Olho/crescimento \& desenvolvimento; Olho/ultra-sonografia; Erros de refração

\section{INTRODUÇ̃̃̃O}

A distribuição dos erros refrativos ao nascimento lembra uma distribuição normal, com pequeno desvio para hipermetropia. O processo envolvido no desenvolvimento ocular (emetropização) produz uma distribuição leptocúrtica, ou seja, uma distribuição onde existe excesso de erros refrativos ao redor da emetropia ${ }^{(1-2)}$.

Estudos in vivo têm reportado distribuição normal dos componentes oculares, distribuição leptocúrtica dos erros refrativos e correlação significante entre o erro refrativo e o comprimento axial ${ }^{(1-3)}$. Estas observações têm levado à hipótese de que os erros refrativos resultem de uma mal combinada associação entre os componentes oculares ${ }^{(1-10)}$.

A evidência de um mecanismo de emetropização no homem foi revisada recentemente por Troilo ${ }^{(11)}$, hipótese ainda não comprovada em experimentos controlados. Mark ${ }^{(12)}$ teorizou que ocorreriam mudanças, principalmente no segmento anterior, representadas pela redução do poder refrativo da 
córnea, aprofundamento da câmara anterior e redução do poder refrativo do cristalino. Van Alphen ${ }^{(13)}$ considerou que o tônus do músculo ciliar e a tensão coroidal influenciariam no aumento do comprimento axial. Haveria ainda a participação de transmissores humorais no processo de emetropização ${ }^{(14)}$.

O crescimento axial do bulbo ocular fica sem controle quando este é ocluído no início da vida sendo privado da formação da imagem na retina. Em animais, a privação da visão causa, geralmente, aprofundamento da câmara vítrea e desvio miópico. Wiesel e Raviola ${ }^{(15)}$ confirmaram estes achados com a oclusão da rima palpebral de macacos recém-nascidos, fato posteriormente confirmado pelos estudos de von Noorden e Crawford ${ }^{(16)}$. Em crianças, alterações na transparência dos meios oculares ou a oclusão unilateral podem causar desenvolvimento de ametropias ${ }^{(17-19)}$.

O processo de emetropização alcança a estabilidade entre 6 e 8 anos de idade ${ }^{(20)}$. Nos primeiros anos da vida, a ocorrência de crescimento não proporcional dos componentes oculares leva à ametropia ${ }^{(21)}$.

Por que alguns indivíduos apresentam anisometropia? A anisometropia pode ocorrer pela ruptura precoce da visão binocular influenciando no desenvolvimento ocular binocular. Ou seja, o processo de emetropização que deveria se estabelecer envolvendo ambos os olhos, perde a sua efetividade $^{(6,22)}$. Pode-se, assim, admitir como hipótese de estudo, que em anisometropia, o olho portador da maior ametropia, ou seja, o que não foi adequadamente estimulado no período crítico do desenvolvimento visual desenvolveu-se sem que os seus componentes oculares apresentassem as mesmas correlações observadas no olho portador da menor ametropia (o que teria sido estimulado adequadamente). Para testar esta hipótese, realizou-se esta investigação com o objetivo de comparar em indivíduos anisométropes, as correlações dos componentes oculares com o erro refrativo total do olho portador da menor e da maior ametropia.

\section{MÉTODOS}

Trata-se de um "survey" analítico, conduzido em população de anisométropes atendidos na Clínica Oftalmológica do Hospital das Clínicas da Faculdade de Medicina da Universidade de São Paulo por demanda espontânea ou referenciada por outros setores do mesmo serviço no período compreendido entre junho e outubro de 2006. Todos os procedimentos aos quais os participantes do estudo foram submetidos tiveram aprovação prévia do Conselho de Ética do Hospital das Clínicas da Faculdade de Medicina de São Paulo, através de revisão do protocolo de pesquisa $\mathrm{n}^{\circ} 109 / 2006$. Antes de serem incluídos neste estudo, os participantes assinaram um termo de consentimento. Foram incluídos anisométropes de 2,00 dioptrias (D) ou mais, calculadas no meridiano vertical. Foram excluídos os anisométropes que apresentavam ectasia corneana, afacia ou pseudofacia, subluxação do cristalino, cirurgia ocular prévia ou doença infecciosa do segmento ocular externo.
Todos os participantes foram submetidos à cicloplegia, instilando-se uma gota em cada olho, três vezes, com intervalos de 10 minutos, do colírio de cloridrato de ciclopentolato a 1\% (Cicloplégico, Laboratório Allergan Produtos Farmacêuticos Ltda). Os exames ceratométrico e refratométrico foram realizados 30 minutos depois, empregando-se o refrator automático Topcon KR 8800, programado para apresentação dos resultados. Foram obtidas três medidas do erro refrativo, e a média foi testada subjetivamente no refrator manual. Em seguida, os componentes oculares, profundidade de câmara anterior, espessura do cristalino, profundidade da câmara vítrea e comprimento axial, foram medidos com o emprego de um biômetro ultra-sônico da marca Tommey, modelo 510, com sonda de freqüência de 10 megaHertz, constante de 118,30 e velocidade do som regulado para $1532 \mathrm{~m} / \mathrm{s}$ para a cavidade vítrea e $1640 \mathrm{~m} / \mathrm{s}$ para o cristalino. O valor de cada componente ocular representou a média de três medidas biométricas. O comprimento do segmento anterior foi obtido pela soma dos valores da profundidade da câmara anterior e da espessura do cristalino. Para o cálculo do poder equivalente do cristalino e do poder refrativo equivalente do olho utilizou-se o método de Bennett ${ }^{(23)}$ que requer dados do erro refrativo, poder da córnea, profundidade da câmara anterior, espessura axial do cristalino e profundidade da câmara vítrea.

Dos 68 anisométropes avaliados, $42(61,76 \%)$ eram do sexo feminino e $26(38,24 \%)$ do sexo masculino. A idade das pessoas incluídas foi de 27,66 $\pm 7,73$ anos, variando entre 9 e 39 anos. Do total, $32(47,06 \%)$ apresentam anisometropia entre $2,00 \mathrm{De} 3,00 \mathrm{D} ; 20(29,41 \%)$ entre $2,01 \mathrm{D}$ e $3,00 \mathrm{D}$ e $16(23,53 \%)$ acima de 3,01 D.

Para a análise dos dados foi criado um banco de dados no Programa Access e empregados os programas Excel e SPSS 10.0 para Windows. A análise dos dados dos olhos portadores do maior e do menor erro refrativo foi feita calculando-se as médias e desvios-padrão dos componentes oculares e a comparação entre os olhos foi feita com o Teste t. Para a análise de correlação entre as variáveis, empregou-se o teste de correlação de Pearson (bi-caudal). Em todos os testes fixou-se em 0,05 ou $5 \%$ o nível para rejeição da hipótese de nulidade, assinalando-se com asterisco, os valores significantes, sendo * para $0,05 \mathrm{e}^{* *}$ para 0,01 .

\section{RESULTADOS}

Os resultados das médias e desvios-padrão dos erros refrativos e dos componentes oculares estão na tabela 1. Os resultados das correlações dos componentes do erro refrativo do olho portador da menor e da maior ametropia estão nas tabelas 2 e 3 .

\section{DISCUSSÃO}

Steiger ${ }^{(24)}$ foi o primeiro a mostrar que o olho emétrope poderia ser construído pela combinação de várias curvaturas 


\begin{tabular}{|c|c|c|}
\hline & $\begin{array}{c}\text { Média e } \\
\text { desvio-padrão }\end{array}$ & $\begin{array}{c}\text { Teste } \\
\text { estatístico }\end{array}$ \\
\hline \multicolumn{3}{|c|}{ Refração do meridiano vertical } \\
\hline Maior ametropia & $-5,46 \pm 7,35(D)$ & \\
\hline Menor ametropia & $-2,78 \pm 4,40(\mathrm{D})$ & $p=0,0111^{*}$ \\
\hline \multicolumn{3}{|c|}{ Poder da córnea médio } \\
\hline Maior ametropia & $43,87 \pm 1,85(D)$ & \\
\hline Menor ametropia & $43,87 \pm 1,48(D)$ & $p=0,9937$ \\
\hline \multicolumn{3}{|c|}{ Profundidade da câmara anterior } \\
\hline Maior ametropia & $3,69 \pm 0,90(\mathrm{~mm})$ & \\
\hline Menor ametropia & $3,74 \pm 0,99(\mathrm{~mm})$ & $p=0,7611$ \\
\hline \multicolumn{3}{|c|}{ Comprimento do segmento anterior } \\
\hline Maior ametropia & $6,21 \pm 0,95(\mathrm{~mm})$ & \\
\hline Menor ametropia & $6,21 \pm 1,03(\mathrm{~mm})$ & $p=0,9698$ \\
\hline \multicolumn{3}{|c|}{ Poder equivalente do cristalino } \\
\hline Maior ametropia & $35,30 \pm 11,40$ (D) & \\
\hline Menor ametropia & $32,25 \pm 9,24$ & $p=0,2524$ \\
\hline \multicolumn{3}{|c|}{ Profundidade da câmara vítrea } \\
\hline Maior ametropia & $15,89 \pm 3,33(\mathrm{~mm})$ & \\
\hline Menor ametropia & $15,58 \pm 2,67(\mathrm{~mm})$ & $p=0,5413$ \\
\hline \multicolumn{3}{|l|}{ Comprimento axial } \\
\hline Maior ametropia & $25,65 \pm 3,13(\mathrm{~mm})$ & \\
\hline Menor ametropia & $24,87 \pm 2,37(\mathrm{~mm})$ & $p=0,1041$ \\
\hline \multicolumn{3}{|c|}{ Poder refrativo equivalente do olho } \\
\hline Maior ametropia & $73,08 \pm 9,87(D)$ & \\
\hline Menor ametropia & $71,38 \pm 8,05(\mathrm{D})$ & $p=0,21715$ \\
\hline
\end{tabular}

corneanas e comprimentos axiais e que os erros refrativos expressavam associações livres dos diferentes componentes oculares da refração. Neste estudo, medimos os componentes oculares da refração de anisométropes e fizemos a comparação entre os olhos portadores da menor e da maior ametropia e não identificamos diferenças significativas entre eles (Tabela 1).

A idéia intrigante de que a modificação de um ou mais destes parâmetros poderia influenciar a refração e que poderiam ser regulados para conseguir a emetropia ganhou força com os estudos conduzidos em gêmeos monozigóticos ${ }^{(25-26)}$. Sabe-se que a hereditariedade determina a tendência de certas proporções destes componentes e que o ambiente influencia o mecanismo da emetropização ${ }^{(27)}$. Pesquisas demonstraram que o olho humano é geneticamente programado para alcançar a emetropia e depois mantê-la no avançar dos anos ${ }^{(21)}$. O mecanismo da emetropização envolve um sistema de retro-alimentação da informação do foco da imagem retiniana e conseqüente ajuste do comprimento axial. A formação de imagem defeituosa interfere neste mecanismo e a ametropia se estabelece. A anisometropia poderia ocorrer da ruptura da visão binocular precoce que influenciaria no desenvolvimento ocular binocular ${ }^{(6,22)}$. Ou seja, o processo de emetropização que deveria estabelecer-se em ambos os olhos, perderia sua efetividade no olho não adequadamente estimulado ${ }^{(6)}$. A maior ametropia deste olho seria o resultado de modificações não proporcionais dos componentes oculares. Em outras palavras, o processo de emetropização é dependente de estímulo visual

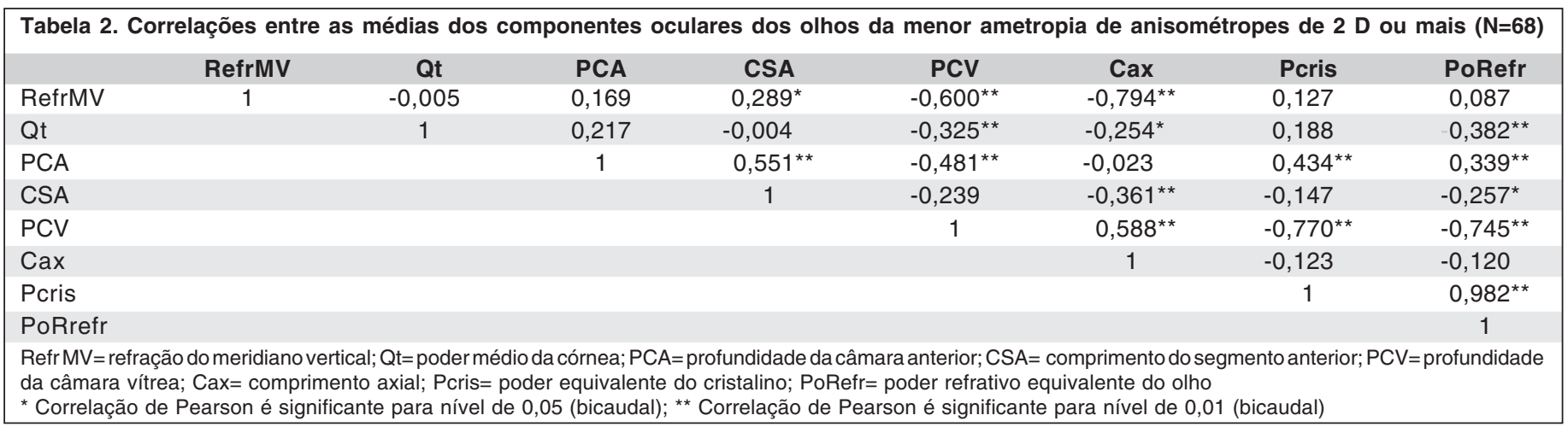

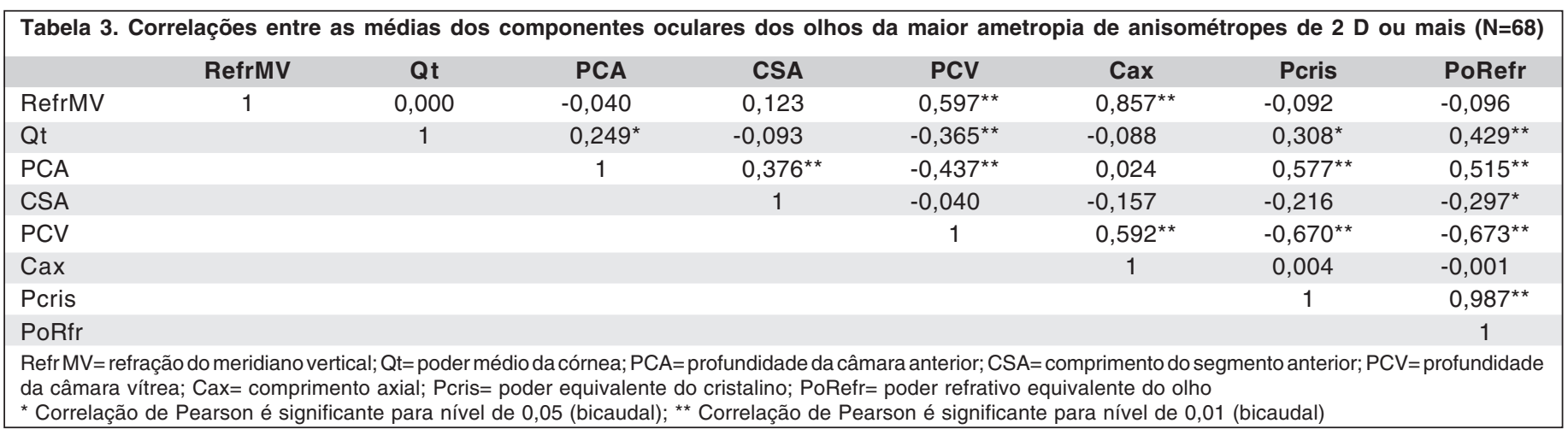


adequado no período crítico de desenvolvimento ocular ${ }^{(22)}$. Miller-Meeks et al. ${ }^{(28)}$ encontraram associação entre miopia unilateral e história de hemorragia vítrea que obscureceu o pólo posterior do bulbo ocular antes da idade de um ano. Os autores teorizaram que a forma de privação da visão induzida pela hemorragia vítrea resultava em alteração do controle do crescimento ocular do olho afetado. Anomalias binoculares associam-se com anisometropia. Abrahamsson et al. ${ }^{(29)}$ observaram que a anisometropia freqüentemente ocorre seguindo o início do estrabismo, por ruptura do processo de emetropização no olho estrábico. Smith et al. ${ }^{(30)}$ induziram cirurgicamente ou opticamente estrabismo em macacos. Encontraram 3\% de macacos anisométropes no grupo controle; $70,8 \%$ de anisométropes no grupo em que o estrabismo foi induzido cirurgicamente e $36 \%$ de anisométropes no grupo em que o estrabismo foi induzido opticamente. Para os autores, o estrabismo altera a coordenação do desenvolvimento ocular binocular, favorecendo o desenvolvimento de anisometropia. As crianças que desenvolvem binocularidade têm ambos os olhos visualmente estimulados. Aquelas que apresentarem ruptura da visão binocular no período crítico de desenvolvimento terão um dos olhos adequadamente estimulado (este estará submetido ao processo de emetropização) e o outro não ${ }^{(29)}$.

Recentemente, Koretz et al. ${ }^{(31)}$ analisaram as correlações entre os vários componentes oculares de olhos emétropes e amétropes. No quadro 1 mostramos os achados de Koretz et al. ${ }^{(31)}$ de olhos emétropes e os comparamos com os olhos portadores da menor ametropia deste estudo. Observamos que as mesmas correlações foram encontradas nas duas situações, reforçando a hipótese de que os olhos portadores da menor ametropia de anisométropes, ou seja, os que supostamente foram mais bem estimulados durante o período crítico do desenvolvimento visual, apresentaram as mesmas correlações encontradas nos olhos emétropes.

No quadro 2 mostramos os achados de Koretz et al. ${ }^{(31)}$ de olhos amétropes e os comparamos com os olhos portadores da maior ametropia deste estudo. Houve concordância nas correlações da refração com o comprimento axial e do comprimento axial com a profundidade da câmara vítrea e na ausência de correlação entre a refração e o poder da córnea.

No quadro 3 comparamos as correlações encontradas neste estudo. Ambos os olhos apresentaram correlação do comprimento axial com a profundidade da câmara vítrea e com a refração, Apresentaram, também, correlação do comprimento do segmento anterior com o poder refrativo equivalente do olho. Não apresentaram correlação do poder da córnea com a refração e com o comprimento do segmento anterior e nem do comprimento do segmento anterior com a profundidade da câmara anterior. Somente os olhos menos amétropes apresentaram correlação entre o poder da córnea e o comprimento axial. Estas alterações embasam a observação de que o aumento proporcional do olho reduz o poder do sistema dióptrico na proporção que aumenta o comprimento axial.

Van Alphen ${ }^{(13)}$ revisou os dados de Stentrön ${ }^{(2)}$ e de Benjamin et al. ${ }^{(32)}$ com o emprego de análise de regressão múltipla e
Quadro 1. Correlações dos componentes oculares em emetropia ${ }^{(31)}$ e em anisometropia (olho portador da menor ametropia)

$\begin{array}{lll} & \text { Emetropia } & \text { Menor ametropia } \\ \text { Refração/Cax } & \text { Correlação } & \text { Correlação } \\ \text { Refração/CSA } & \text { Correlação } & \text { Correlação } \\ \text { Refração/Qt } & \text { Não correlação } & \text { Não correlação } \\ \text { Cax/Qt } & \text { Correlação forte } & \text { Correlação } \\ \text { Cax/PCV } & \text { Correlação fraca } & \text { Correlação } \\ \text { CSA/Qt } & \text { Não correlação } & \text { Não correlação } \\ \text { CSA/PCV } & \text { Não correlação } & \text { Não correlação } \\ \text { CSA/PoRefr } & \text { Correlação } & \text { Correlação }\end{array}$

$\mathrm{Cax}=$ comprimento axial; $\mathrm{CSA}=$ comprimento do segmento anterior; $\mathrm{Qt}=$ poder médio da córnea; $\mathrm{PCV}=$ profundidade da câmara vítrea; $\mathrm{PoRefr}=$ poder refrativo equivalente do olho

\begin{tabular}{|c|c|c|}
\hline & Ametropia & Maior ametropia \\
\hline Refração/Cax & Correlação & Correlação \\
\hline Refração /CSA & Correlação & Não correlação \\
\hline Refração/Qt & Não correlação & Não correlação \\
\hline $\mathrm{Cax} / \mathrm{Qt}$ & Correlação fraca & Não correlação \\
\hline Cax/PCV & Correlação & Correlação \\
\hline CSA/Qt & Correlação & Não correlação \\
\hline CSA/PCV & Correlação & Não correlação \\
\hline CSA/PoRefr & Não correlação & Correlação \\
\hline \multicolumn{3}{|c|}{$\begin{array}{l}\text { Cax = comprimento axial } ; \mathrm{CSA}=\text { comprimento do segmento anterior; } \mathrm{Q}=\text { poder } \\
\text { médio da córnea; } \mathrm{PCV}=\text { profundidade da câmara vítrea; } \mathrm{PoRefr}=\text { poder refrativo } \\
\text { equivalente do olho }\end{array}$} \\
\hline
\end{tabular}

\begin{tabular}{|c|c|c|}
\hline \multicolumn{3}{|c|}{$\begin{array}{l}\text { Quadro 3. Correlações dos componentes oculares em anisometropia. } \\
\text { São Paulo, } 2006\end{array}$} \\
\hline & Menor ametropia & Maior ametropia \\
\hline Refração/Cax & Correlação & Correlação \\
\hline Refração/CSA & Correlação & Não correlação \\
\hline Refração/Qt & Não correlação & Não correlação \\
\hline Cax/Qt & Correlação & Não correlação \\
\hline Cax/PCV & Correlação & Correlação \\
\hline CSA/Qt & Não correlação & Não correlação \\
\hline CSA/PCV & Não correlação & Não correlação \\
\hline CSA/PoRefr & Correlação & Correlação \\
\hline \multicolumn{3}{|c|}{$\begin{array}{l}\text { Cax }=\text { comprimento axial; } \mathrm{CSA}=\text { comprimento do segmento anterior; } \mathrm{Qt}=\text { poder } \\
\text { médio da córnea; } \mathrm{PCV}=\text { profundidade da câmara vítrea; } \mathrm{PoRefr}=\text { poder refrativo } \\
\text { equivalente do olho }\end{array}$} \\
\hline
\end{tabular}

concluiu que as inúmeras correlações apresentadas eram essencialmente o resultado de poucas variáveis atuando independentemente, e sugeriu que para olhos emétropes, no mínimo dois fatores independentes eram relevantes.

$\mathrm{O}$ primeiro fator, denominado de $\mathrm{S}$, foi considerado determinante da relação do poder corneano com o comprimento axial. Para van Alphen ${ }^{(13)}$ resultaria do fator $\mathrm{S}$ uma tendência de olhos mais compridos apresentarem córneas mais planas, independentemente do erro refrativo. Neste estudo, encontramos correlação do comprimento axial com o poder corneano 
somente nos olhos portadores da menor ametropia. Para Koretz et al. ${ }^{(31)}$ o comprimento axial correlaciona-se com o poder corneano em olhos emétropes e amétropes, porém, de forma fraca em olhos amétropes.

$\mathrm{O}$ segundo fator, denominado de $\mathrm{P}$, agrupava comprimento axial, poder do cristalino e profundidade da câmara anterior. Para van Alphen ${ }^{(13)} \mathrm{o}$ fator $\mathrm{P}$ representaria a tendência de olhos mais compridos apresentarem câmaras anteriores mais profundas e cristalinos mais planos. Como no estudo de Koretz et al. ${ }^{(31)}$ evidenciamos correlação do comprimento axial com a profundidade da câmara vítrea em ambos os olhos e correlação do comprimento do segmento anterior com o erro refrativo apenas nos olhos portadores da menor ametropia. Ainda em ambos os olhos, evidenciamos correlação do poder do cristalino com a profundidade da câmara anterior e com o poder refrativo equivalente do olho. O poder efetivo do cristalino é reduzido pelo aprofundamento da câmara anterior.

Contudo, em relação à ametropia, van Alphen ${ }^{(13)}$ introduziu um terceiro fator que denominou de R. O fator R foi associado à resistência à pressão ocular oferecida pela coróide/ músculo ciliar. O grau de tensão coroidal influenciaria na determinação do comprimento axial. Van Alphen ${ }^{(13)}$ propôs que o processo de emetropização ocorreria por um mecanismo de controle de autofoco por retro-alimentação negativo. Variações no tônus do músculo ciliar poderiam produzir alterações refrativas por interferirem no mecanismo de auto-foco. Dessa forma, olhos de quaisquer tamanhos (fator $\mathrm{S}$ ), hipermétropes ao nascimento, teriam que alongar (fator $\mathrm{P}$ ) para se tornarem emétropes. Nesse processo o comprimento axial seria ajustado pelo poder refrativo total. $\mathrm{O}$ grau de ajustamento (fator $\mathrm{R}$ ) determinaria a refração e a forma do bulbo ocular. Ou seja, o fator $\mathrm{R}$ representaria o grau de ametropização ou de ajustamento do fator P com respeito ao fator S. Para Koretz et al. ${ }^{(31)}$, as diferentes correlações observadas nos olhos emétropes e amétropes reforçariam o conceito de que o olho amétrope falhou em crescer com proporcionalidade.

Neste estudo conduzido em anisométropes verificamos que o olho portador da menor ametropia, que poderia ter sido o olho mais bem estimulado durante o período crítico de desenvolvimento visual, apresentou as correlações mais freqüentemente observadas nos olhos emétropes. Em contrapartida, os olhos adelfos, que poderiam não ter recebido a mesma estimulação visual, não desenvolveram as correlações mais frequientemente observadas nos olhos emétropes, tornandose, portanto os olhos mais amétropes.

\section{ABSTRACT}

Purpose: To asses the correlation between ocular components (axial length, anterior segment length, corneal power, vitreous length and equivalent power of the eye) and refractive error in eyes with higher and lower ametropia of subjects with anisometropia. Methods: An analytical survey was carried out in 68 patients with anisometropia of two or more diopters, assisted at the Ophthalmological Clinic from "Hospital das Clínicas da Faculdade de Medicina" of São Paulo University. All participants were submitted to ophthalmologic examination which included objective and subjective cycloplegic refratometry, keratometry and ultrasonic biometry. Results: No significant difference between measures of ocular components of the eyes with higher and lower ametropias was observed. The eyes with lower ametropia presented the same significant correlations observed in emmetropic eyes: of refraction with anterior segment length and axial length, and of the axial length with corneal power and vitreous length. The eyes with higher ametropia presented significant correlation of refraction with the axial length and of the axial length with vitreous length. Furthermore, we observed in both eyes, significant correlation of the power of the crystalline lens with the anterior chamber depth. Conclusion: The eyes with lower ametropia presented correlations more frequently observed in emmetropia. The eyes with higher ametropia did not present the same correlations observed in emmetropic eyes.

Keywords: Anisometropia; Biometry/methods; Eye/ultrasonography; Eye/growth \& development; Refractive errors

\section{REFERÊNCIAS}

1. Sorsby A, Benjamin B, Sheridan M, Stone J, Leary GA. Refraction and its components during the growth of the eye from the age of three. Memo Med Res Counc. 1961;301(Special):1-67.

2. Stenström S. Investigation of the variation and correlation of the optical elements of human eyes. Am J Optom Arch Am Acad Optom. 1948;48:218-32.

3. Sorsby A. Biology of the eye as an optical system. In: Tasman W, Jaeger EA, editors. Duane's clinical ophthalmology. Philadelphia: Lippincott-Raven; 1995. v.1. Cap.34. p.1-17.

4. Fledelius HC. Ophthalmic changes from age of 10 to 18 years. A longitudinal study of sequels to low birth weight. III. Ultrasound oculometry and keratometry of anterior eye segment. Acta Ophthalmol (Copenh). 1982;60(3):393-402.

5. Fledelius HC. Ophthalmic changes from age of 10 to 18 years. A longitudinal study of sequels to low birth weight. IV. Ultrasound oculometry of vitreous and axial length. Acta Ophthalmol (Copenh). 1982;60(3):403-11.

6. Giovedi Filho R, Alves MR, Giovedi MRA, Shato CWM, Netto AL. Componentes ópticos refrativos em anisometropia. Rev Bras Oftalmol. 2003;62(3):175-8.

7. Garner LF, Kinnear RF, McKellar M, Klinger J, Hovander MS, Grosvenor T. Refraction and its components in Melanesian schoolchildren in Vanuatu. Am J Optom Physiol Opt. 1988;65(3):182-9.

8. Larsen JS. The sagittal growth of the eye. II. Ultrasonic measurement of the axial diameter of the lens and the anterior segment from birth to puberty. Acta Ophthalmol (Copenh). 1971;49(3):427-40.

9. Larsen JS. The sagittal growth of the eye. 3. Ultrasonic measurement of the posterior segment (axial length of the vitreous) from birth to puberty. Acta Ophthalmol (Copenh). 1971;49(3):441-53.

10. Tokoro T, Suzuki K. Changes in ocular refractive components and development of myopia during seven years. Jpn J Ophthalmol. 1969;13:27-34.

11. Troilo D. Neonatal eye growth and emmetropisation - a literature review. Eye. 1992;6(Pt 2):154-60. Review.

12. Mark HH. Emmetropization. Physical aspects of a statistical phenomenon. Ann Ophthalmol. 1972;4(5):393-4 passim.

13. Van Alphen G. On emmetropia and ametropia. Opt Acta (Lond). 1961;142 (Suppl):1-92.

14. Smith EL $3^{\text {rd }}$, Fox DA, Duncan CC. Refractive-error changes in kitten eyes produced by chronic on-channel blockade. Vision Res. 1991;31(5):833-44.

15. Wiesel TL, Raviola E. Myopia and eye enlargement after neonatal lid fusion on monkeys. Nature. 1977;266(5597):66-8.

16. Von Noorden GK,Crawford ML. Lid closure and refractive error in macaque monkeys. Nature. 1978;272(5648):53-4. 
17. Hoyt CS, Stone RD, Fromer C, Billson FA. Monocular axial myopia associated with neonatal eyelid closure in human infants. Am J Ophthalmol. 1981;91(2):197-200.

18. Gee SS, Tabbara KF. Increase in ocular axial length in patients with corneal opacification. Ophthalmology. 1988;95(9):1276-8.

19. Giovedi Filho R, Alves MR, Giovedi MRA, Netto AL, Pwa HWT. Características de uma população de escolares anisométropes. Rev Bras Oftalmol. 2003;62(2):103-7.

20. Gwiazda J, Thorn F, Bauer J, Held R. Emmetropization and the progression of manifest refraction in children followed from infancy to puberty. Clin Vis Sci. 1993;8:337-44

21. Brown PN, Koretz JF, Bron AJ. The development and maintenance of emmetropia. Eye. 1999;13(Pt 1):83-92.

22. Smith EL $3^{\text {rd }}$, Harwerth RS, Crawford ML, von Noorden GK. Observations on the effects of form deprivation on the refractive status of the monkey. Invest Ophtalmol Vis Sci. 1987;28(8):1236-45.

23. Bennett AG. A method of determining the equivalent powers of the eye and its crystalline lens without resort to phakometry. Ophthalmic Physiol Opt. $1988 ; 8(1): 53-9$
24. Steiger A. Die Entstehung der spharischen. Refraktionen des menschlichen Auges. Berlin: Karger, 1913

25. Sorsby A, Sheridan M, Leary GA. Refraction and its components in twins Spec Rep Ser Med Res Counc (GB). 1962;(303):1-43.

26. Kimura T. Development changes of the optical components in twins. Acta Soc Ophthalmol Jpn. 1965;69:963-9.

27. Norton TT, Siegwart JT Jr. Animal models of emmetropization: matching axial length to the focal plane. J Am Optom Assoc. 1995;66(7):404-14.

28. Miller-Meeks MJ, Bennett SR, Keech RV, Blodi CF. Myopia induced by vitreous hemorrhage. Am J Ophthalmol. 1990;109(2):199-203.

29. Abrahamsson M, Fabian G, Sjostrand J. Refraction changes in children developing convergent or divergent strabismus. Br J Ophthalmol. 1992;76(12):723-7.

30. Smith EL $3^{\text {rd }}$, Hung LF, Harwerth RS. Effects of optically induced blur on the refractive status of young monkeys. Vision Res. 1994;34(3): 293-301.

31. Koretz JF, Rogot A, Kaufman PL. Physiological strategies for emmetropia. Trans Am Ophthalmol Soc. 1995;93:105-18; discussion 118-22.

32. Benjamin B, Davey JB, Sheridan M, Sorsby A, Tanner JM. Emmetropia and its aberrations; a study in the correlation of the optical components of the eye. Spec Rep Ser Med Res Counc (GB). 1957;11(293):1-69.

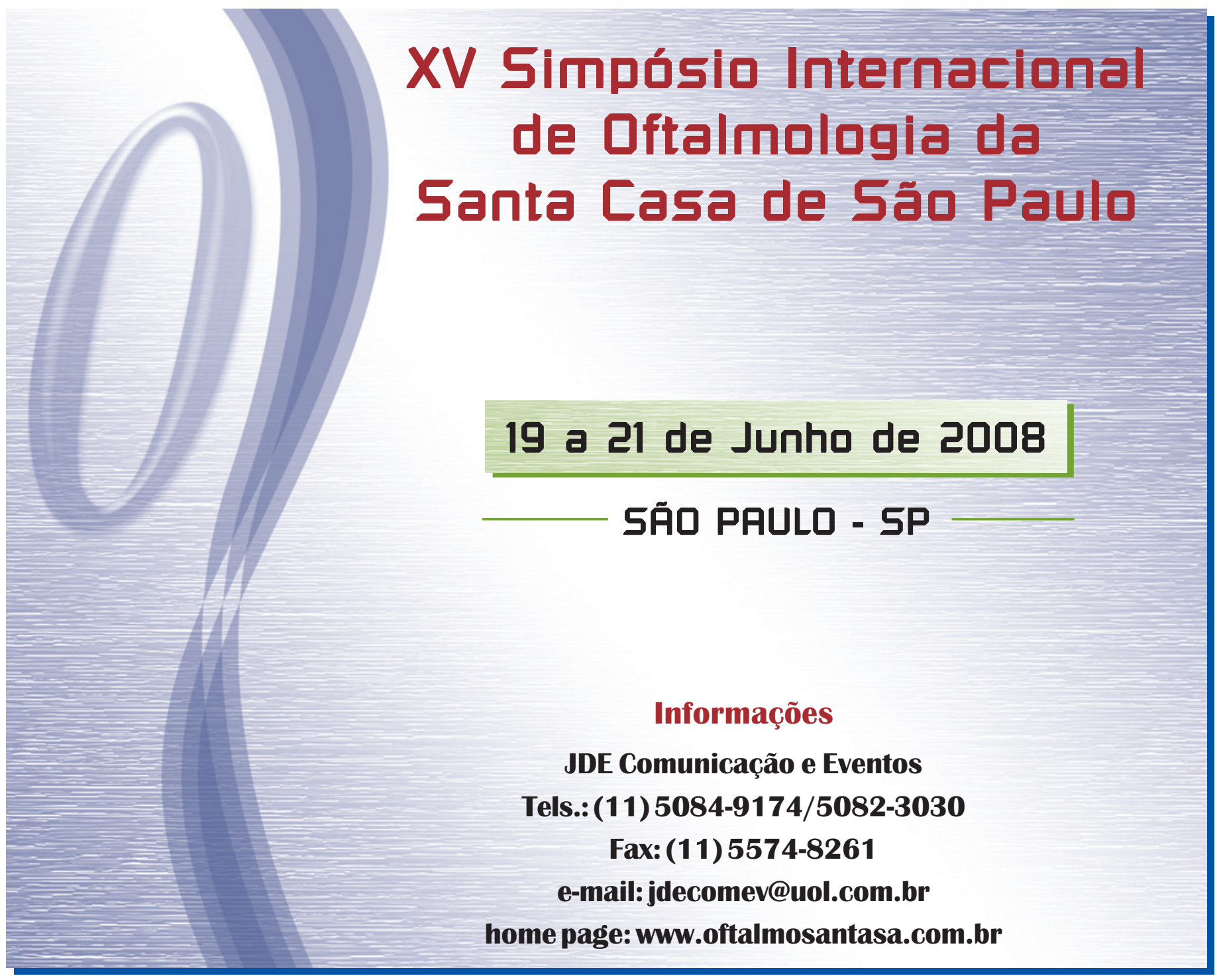

